\title{
Transcatheter Aortic Valve Implantation (TAVI): Is General Anaesthesia a Necessity?
}

\author{
Sugeevan Savarimuthu ${ }^{1}$ and Amer Harky ${ }^{2}$ \\ ${ }^{1}$ Broomfield Hospital \\ ${ }^{2}$ Liverpool Heart and Chest Hospital NHS Foundation Trust
}

July 26, 2021

\begin{abstract}
Transcatheter Aortic Valve Implantation (TAVI) has emerged over the last decade and has become a viable treatment option for those with severe symptomatic aortic stenosis (AS). The numbers of TAVI that are being performed in centres across the world is rising rapidly, and revolutionising treatment for AS and steering away from the need for open heart surgery. TAVI has been targeted for those who are deemed high risk to undergo open heart surgery such as the elderly, frail, or those with multiple co-morbidities. General anaesthesia (GA) has been used to facilitate TAVI especially when the procedure was in its infancy. The use of GA comes with its own set of risks such as haemodynamic compromise, risk for aspiration, longer procedure time and post-operative nausea and vomiting. These side effects and or complications may not be tolerated well in those who are elderly or have numerous co-morbidities. This article seeks to understand the outcomes of patients who undergo TAVI with the support of sedation and local anaesthesia (LA) in comparison to those who received GA.
\end{abstract}

Transcatheter Aortic Valve Implantation (TAVI): Is General Anaesthesia a Necessity?

Sugeevan Savarimuthu MD MRCP [1], Amer Harky MBChB, MRCS, MSC [2]

1) Department of Medicine, Broomfield hospital, Chelmsford, UK

2) Faculty of Cardiothoracic surgery, Liverpool Heart and Chest Hospital, Liverpool, UK

Abstract

Transcatheter Aortic Valve Implantation (TAVI) has emerged over the last decade and has become a viable treatment option for those with severe symptomatic aortic stenosis (AS). The numbers of TAVI that are being performed in centres across the world is rising rapidly, and revolutionising treatment for AS and steering away from the need for open heart surgery. TAVI has been targeted for those who are deemed high risk to undergo open heart surgery such as the elderly, frail, or those with multiple co-morbidities. General anaesthesia (GA) has been used to facilitate TAVI especially when the procedure was in its infancy. The use of GA comes with its own set of risks such as haemodynamic compromise, risk for aspiration, longer procedure time and post-operative nausea and vomiting. These side effects and or complications may not be tolerated well in those who are elderly or have numerous co-morbidities. This article seeks to understand the outcomes of patients who undergo TAVI with the support of sedation and local anaesthesia (LA) in comparison to those who received GA.

\section{Declaration:}

International review board, consent statement and clinical trial registration are not applicable to this study

\section{Key words}


Transcatheter aortic valve implantation, TAVI, General anaesthesia, Sedation, Local anaesthesia

Abbreviations

AS - Aortic Stenosis

AR - Aortic regurgitation

BIS - Bispectral index

BMI - Body mass index

CS - Conscious sedation

CVC - Central venous catheterisation

DS - Deep sedation

GA - General anaesthesia

ICU - Intensive care unit

LA - Local anaesthesia

LogEuroSCORE - Logistic European System for Cardiac Operative Risk Evaluation

LVEF - Left ventricular ejection fraction

MAP - Mean arterial pressure

NYHA - New York Heart Association

POD - Post operative delirium

PVL - Paravalvular leak

TA - Transapical

TAVI - Transcatheter aortic valve implantation

TF - Transfemoral

TIVA - Total intravenous anaesthesia

TOE - Transoesophageal echocardiography

TTE - Transthoracic echocardiography

Introduction

Transcatheter Aortic Valve Implantation (TAVI) has emerged over the last decade and has become a viable treatment option for those with severe symptomatic aortic stenosis (AS). The numbers of TAVI that are being performed in centres across the world is rising rapidly, and revolutionising treatment for AS and steering away from the need for open heart surgery. TAVI has been targeted for those who are deemed high risk to undergo open heart surgery such as the elderly, frail, or those with multiple co-morbidities. General anaesthesia (GA) has been used to facilitate TAVI especially when the procedure was in its infancy. The use of GA comes with its own set of risks such as haemodynamic compromise, risk for aspiration, longer procedure time and post-operative nausea and vomiting. These side effects and or complications may not be tolerated well in those who are elderly or have numerous co-morbidities.

Choice of GA, Sedation and/or LA for patients planned for TAVI should be discussed in a multidisciplinary team meeting including cardiologists, cardiac surgeons, anaesthetists, radiologists, and clinical nurse specialists. Factors such as age, co-morbidities, vascular access and anaesthetic fitness and risk are all vital in 
assessing whether a patient can undergo a GA. Patients with advanced age, frailty, significant lung disease or severe co-morbidities may be challenging to anaesthetise. Cardiopulmonary exercise testing, up to date lung function tests, and pre assessment clinics can aid to filter these patients. Vascular access such as poor femoral artery access can affect the operators experience and may affect how the anaesthetic is performed such as in cases of transcarotid TAVI. Sedation and LA should be tolerated well in most patients even those who may be restless or anxious. Centre and operator experience can guide decision making and the presence of local cardiac surgery support is paramount. LogEuroSCORE can be used to assess the operative mortality in those undergoing cardiac surgery and may be used for TAVI procedure, with higher values associated with increased GA risk. [1]

Table 1 shows a breakdown of the LogEuroSCORE.

Patient Selection and Choice of Access

Selecting the right group of patients to undergo TAVI can be challenging. Those who have been referred for a TAVI are already considered a high surgical risk and encompass features which are difficult to anaesthetist. Being able to determine those who can undergo GA and those who can't is complex, and the approach must be adapted to each patient. When evaluating patients detailed physical examination, up to date blood tests and appropriate imaging such as TTE, baseline electrocardiogram and 24-hour tape, and cardiac catheterisation needs to be performed to ensure fitness for the procedure. Pulmonary function tests and cardiopulmonary exercise testing (CPEX) can aide in assessment of cardiovascular and respiratory function and can help quantify general anaesthetic risk. Scoring systems such as logEuroSCORE II, National surgical quality improvement program (NSQIP), Society of thoracic surgeons (STS) risk score can help determine cardiac operative risk which may be transferred over to assess anaesthetic risk. Those who are elderly, and frail will need to be assessed for functional status and care support which can factor into the decision to use sedation rather than GA. Patients with co-morbidities such as heart failure, chronic kidney disease, history of stroke, chronic obstructive pulmonary disease, and atrial fibrillation will not be able to tolerate periods of hypotension and hypoperfusion which can arise with the use of anaesthetic agents, which may require adjustment of anaesthetic approach or adjusted of doses of medications given. Conversely, they may be sensitive to fluid resuscitation, inotrope and vasopressor use, and hypothermia. The decision between GA and Sedation will ultimately be down to the clinician based on their experience and weighing up the risks and benefits of each technique along with the severity of AS, symptomology and associated co-morbidities and whether the intervention will improve the patient's quality of life. $[2,3]$

Transfemoral approach is typically the preferred route for TAVI, in some cases due to peripheral vascular disease the patient may not have suitable anatomy for the procedure. There are several approaches that can be considered such as trans-axillary, subclavian, transapical. Each comes with its own set of risks which the anaesthetist and cardiologist need to be mindful of such as vascular injuries (e.g., subclavian approach), or lung injury and cardiac tamponade (e.g., transapical). [2]

When using a transcarotid approach, thorough carotid artery assessment prior to the procedure is required to ensure that there is no evidence of significant stenosis, plaque burden or anatomical variation. Anaesthetists must be wary of the risk of cerebral hypoperfusion and embolus formation during the procedure, which can be minimised using transcranial doppler and magnetic resonance angiography. Debry et al studied the outcomes of those who received transcarotid TAVI with the use of lidocaine as LA along with an infusion of remifentanyl as CS. Cerebral perfusion was monitored with cerebral oximetry. A total of 174 patients were included with 52 in the CS group and 122 in the GA group. Mean age was 80.5+/-7.9 years with patients in the CS group more likely to have a history of coronary artery disease and revascularisation. $7.6 \%$ were converted to GA due to discomfort and respiratory distress. Successful carotid vascular access was gained in all cases and device success was achieved in $88 \%$ of the CS group and $93 \%$ of the GA group. There were no cerebrovascular (CVA) or adverse events. There were 3 procedural deaths, and 30-day mortality was $7 \%$. There was no difference in mortality according to the anaesthetic strategy. Post procedural AR was noted in $8.6 \%$ but was not significantly different between the groups. $5.7 \%$ developed CVA during 30 days with all events occurring in the GA group. [4] 
A transapical (TA) approach can also be considered in those who have difficult peripheral vascular access or those who have significant plaque burden making a transcarotid approach difficult. Gauthier et al compared the outcomes of patients undergoing TAVI either via TF or TA under GA or LA+/-Sedation. The choice of agents for GA was left at the anaesthetist's discretion. Propofol infusion or sevoflurane were used for maintenance of anaesthesia. LA was performed by the interventionalist with sedation only used if required. Patients in the TA group had a higher logEuroSCORE, they also stayed significantly longer in ICU and hospital compared to the TF group. Patients who had TF TAVI with sedation had shorter stays than those who had GA. Intrahospital and 30-day mortality was statistically significantly higher in the TA group. 30-day mortality was $17 \%$ in the TA group, $2 \%$ in the TF group under GA and $5 \%$ in the TF group under sedation. Major cardiac complications occurred more frequently in the TA group. One year mortality was calculated to be $24 \%, 11 \%$, and $16 \%$ in the TF GA, TF Sedation, and TA group respectively. Overall estimated survival at maximal follow up was $26 \%$ for the TF GA group (46 months), $63 \%$ for the TF Sedation group (29 months), and $0 \%$ for the TA group (55 months). [5]

\section{Outcomes in General Anaesthesia versus Sedation}

Levels of sedation can vary with the drugs being used, the response of those drugs by the patients, their comorbidities, and lifestyle/social history such as alcohol use. Sedation can range from minimal to conscious to deep should be performed by a clinician experienced in the use and monitoring of patients under anaesthesia.

Table 2 shows the American Society of Anaesthesiologists Definition of General Anaesthesia and Levels of Sedation/Analgesia

Renner et al studied the outcomes of patients at a single centre undergoing TAVI with either GA or Conscious sedation (CS) with anaesthesia performed by two experienced cardiac anaesthesiologists. Both groups used low dose midazolam IV (intravenous) at $0.01-0.02 \mathrm{mg} / \mathrm{kg}$ prior to the procedure. Both groups had an arterial line and central venous catheter. Patients in the GA groups had either an endotracheal intubation or a laryngeal mask airway (LMA). Those who had an endotracheal intubation were able to have transoesophageal echocardiography (TOE), whereas those with a LMA and in the CS group had transthoracic echocardiography (TTE) before and after the procedure. Total intravenous anaesthesia (TIVA) was used in the GA group with either a bolus of propofol or etomidate on induction followed by continuous infusion of propofol $(3-5 \mathrm{mg} / \mathrm{kg} / \mathrm{hr})$ and remifentanyl $(0.3-0.4 \mathrm{mcg} / \mathrm{kg} / \mathrm{min})$ and rocuronium as a muscle relaxant. The CS group used much lower doses of propofol $(0.3-0.5 \mathrm{mg} / \mathrm{kg})$ and remifentanyl $(0.02-0.06 \mathrm{mcg} / \mathrm{kg} / \mathrm{min})$ and $5-10 \mathrm{ml}$ of $1 \%$ Mepivicaine was infiltrated to the groin at the site of vascular access. 107 patients were in the GA group with a mean age of 82 (6.1) years, and 93 patients in the CS group with a mean age of 82 (6.4). There was no difference between groups in terms of co-morbidities, New York Heart Association (NYHA) classification or LogEuroSCORE. Conversion from CS to GA was needed in 5 cases: 4 due to procedural complications and 1 due to agitation. Mean anaesthesia time was significantly longer in the GA group, as expected more anaesthetic agents such as propofol was used up in the GA group and so the need to reach haemodynamic stability with the use of vasopressors and IV fluids were more common in the GA group. Lower mean arterial pressure (MAP) was noted in the GA group. Shorter intensive care unit (ICU) stays were noted in the CS group, though there was no difference in length of stay in hospital between the groups. There was no significant different in 30-day mortality between the groups. Even in those who were high risk with a LogEuroSCORE $>22$ there was no difference in intra-procedural characteristics between groups. $9.3 \%$ patients all of whom were in the GA group required cardiopulmonary resuscitation. [6]

Husser et al studied the outcomes of patients receiving TAVI with the support of either GA or LA or CS (LACS). 16543 patients from multiple centres across Germany were enrolled, mean age was 81 years and LACS was used as the primary approach in $49 \%$ of cases. LACS was more likely to be conducted in experienced TAVI centres. Procedural success was achieved in 97.5\%. The LACS group showed shorter procedural duration and fluoroscopy time. Conversion to sternotomy and bleeding complications were less frequent in the LACS group, though vascular complications were higher. Rate of device embolization and malposition was less in the LACS group. There was no difference in paravalvular leak (PVL) between groups. Post procedural course was less complicated in the LACS group such as delirium, respiratory failure, CPR. 
Duration of ICU and hospital stay was shorter in the LACS group in population matched analysis. 30-day mortality was less in the LACS group, though there was no significant difference at 1 year mortality. [7] Miles et al in a single centre study looked at the outcomes of GA and CS in patients enrolled for TAVI. CS involved a remifentanyl infusion at $0.05 \mathrm{mcg} / \mathrm{kg} / \mathrm{min}$ with a fascia iliaca block ilioinguinal block using 2 $\mathrm{mg} / \mathrm{kg} 0.25 \%$ levobupivacaine. 118 patients were involved with 44 receiving CS. There was a $17 \%$ reduction in anaesthetic time and a $29 \%$ reduction in recovery room time in the CS group. Reduction in inotrope requirement was seen in the CS group and no difference were seen in terms of postprocedural complications between groups. $7 \%$ from the CS group required ICU admission with one due to respiratory failure needing tracheal intubation. $14 \%$ in the GA group need ICU admission. 4 patients required conversion to GA. [8] Goren et al in a single centre study involving 204 patients undergoing TAVI found significantly less use of catecholamines and intravenous fluids in patients received sedation over GA. Total procedural times were also less. Conversion to GA occurred in 4.6\%. In hospital mortality and total length of stay were similar between groups. [9]

Thiele et al performed a multi-centre randomised trial (SOLVE trial) of patient undergoing TAVI with either GA or CS with LA. Patient included had severe AS, age $>75$ years and high risk for surgical valve replacement. GA was provided via TIVA with continuous infusion of propofol and remifentanyl maintaining a BIS of 40-60. LA was provided by the interventionalist. CS was given either by dexmedetomidine, propofol or other nonbenzodiazepine drugs and titrated to keep BIS $>70$. Ventilation was monitored with capnography and supplemental oxygen was given. 225 patients were randomly assigned to the GA group and 222 to CS. Mean age was $81.6+/-5.5$ years, a median logEuroSCORE of $14.8 \%$ and similar baseline characteristics between groups. Conversion to GA from CS occurred in 5.9\% with the reasons being the need for CPR, respiratory insufficiency, and agitation. Minimum oxygen saturation during anaesthesia and cerebral oxygen saturation at the end of the procedure was significantly lower in the CS group. As expected, the quantity of anaesthetic agents used were greater in the GA group. At 30 days the rate of the composite primary end point of all cause mortality, stroke, myocardial infraction, acute kidney injury was similar between groups. Device time, procedural time, POD, moderate to severe prosthetic valve regurgitation at 30 days and cardiovascular mortality did not differ significantly between groups. Mean length of ICU and hospital stays were similar. The need for vasopressor and inotropes were greater in the GA group. [10]

\section{Outcomes with Local Anaesthesia}

The development of LA blocks has provided an avenue for patients with high anaesthetic risks to have effective and long-lasting analgesia and anaesthesia and allowing the operator to perform their procedure while avoiding the risk associated with GA. Romano et al conducted a study on patients who were enlisted for a TAVI and compared the outcomes of Deep sedation (DS) against LA which were performed by interventional cardiologists. 50 patients had a femoral nerve block using ultrasound guidance with $1 \%$ lidocaine and $0.5 \%$ ropivicaine (10-12ml). 32 patients had DS with infusions of propofol $(1-2 \mathrm{mg} / \mathrm{kg} / \mathrm{hr})$ and remifentanyl $(0.1 \mathrm{mcg} / \mathrm{kg} / \mathrm{min})$. Both groups had no differences in baseline characteristics such as NYHA class, Left ventricular ejection fraction (LVEF), LogEuroSCORE II, and co-morbidities. Mean age in the LA group was $85.3+/-4.9$ years and $84 / 2+/-5.7$ in the DS group. $69.5 \%$ had balloon valvuloplasty prior to prosthetic valve implantation and $89 \%$ had a successful TAVI procedure. The rate of periprocedural complications did not differ between groups with the most common being minor vascular complications $20.7 \%$ in the LA group and $22 \%$ in the DS group and pacemaker implantation (14\% in the LA group and $16 \%$ in the DSD group). Haemodynamic monitoring during the procedure revealed differences in the groups. With the DS group having more pronounced reduction in systolic (SBP) and diastolic blood pressure (DBP) throughout the procedure. After TAVI cardiac output rose in the LA group and stroke volume rose in both groups. Systemic vascular resistance (SVR) reduced in all stages of the procedure in the DS group whereas in the LA group SVR reduced at baseline and at the end of the procedure. Global cardiovascular performance was evaluated by looking at cardiac cycle efficiency and found improved performance in the DS group after anaesthesia and improved performance in the LA group after TAVI. The MAP-dicrotic pressure reduced in both groups but more significantly in the DS group. There were 32 cases of aortic regurgitation following TAVI with patients in the LA groups (23) being detected more easily given their stable haemodynamic 
status. [11]

Durand et al studied the outcomes of TAVI in 151 patients with the use of LA alone. They suggest due to reduction in femoral sheath sizes, advances with prosthetic valves and increased operators, what was once performed with GA can be done with LA. Lidocaine $2 \%$ (20 to $30 \mathrm{mls}$ ) was infiltrated to the skin overlying the femoral artery, additional small doses of midazolam and nalbuphine used for sedation if required. SAPIEN and SAPIEN XT valves were used with valve position for these cases were confirmed by using fluoroscopy. Mean LogEuroSCORE was $22.8 \%$ and mean age was $83.3+/-6.4$. Insertion of the SAPIEN valve required a surgical arterial cut down approach and required the presence of a cardiac surgeon, the SAPIEN XT valve was percutaneous. Overall, the procedure was well tolerated with a $95.4 \%$ success rate. Procedural failure was related to unsuccessful vascular access $(2 \%)$ or severe AR (2.6\%). Conversion to GA occurred in $3.3 \%$ (only SAPIEN cases) with only one case needing conversion prior to the procedure due to annulus rupture. The majority were haemodynamically stable with only $5.5 \%$ requiring vasopressors. Endotracheal intubation was not required to improve patient's compliance or comfort. [12]

Oguri et al performed an analysis of the FRANCE 2 registry which compared data from multiple centres and the clinical outcomes of those who had GA and LA for TAVI. 2326 patients of which 1377 had GA and 949 had LA with a mean age of 83.1+/-7.2 years were studied. High success rates were seen in both forms of anaesthesia: $97.6 \%$ in GA and $97 \%$ in LA. TOE as expected was used more often in the GA group. The Edwards valve was used more frequently with the GA group. Length of ICU and hospital stay was greater in the LA group. No differences were noted between groups in terms of complications such as stroke, myocardial infarction, or bleeding complications. Mild post procedural AR was not significantly different between groups, though the incidence was higher in the LA group. $15.1 \%$ of patients died during follow up with $53.1 \%$ of deaths within 30 days. 30-day mortality rates due to respiratory failure had a higher incidence in the GA group. No significant difference was noted between groups in 30 day or 1 year mortality. [13]

Table 3 consists of a summary of current literature on outcomes in TAVI related to anaesthetic choice.

\section{A Case for Transthoracic echocardiography}

The use of TOE had been paramount in the early stages of TAVI use with aiding to observe catheter positions, valve sizing, evaluation of stent position and ruling out any complications from the procedure. The most important use for TOE is to rule out PVL. The occurrence of PVL is reducing at TAVI experience increases and technology has improved. With the shift from GA to sedation techniques, TOE cannot be used in these group of patients and so TTE may provide sufficient information to the operator without having the risks of a GA or TOE intubation of the oesophagus. Jain et al observed the outcomes and findings of those who has TTE during TAVI. 278 patients were included in the study and received sedation. Mean age was $82.1+/-7.3$ years, with $52.5 \%$ of female patients. There were no intraprocedural deaths. $1.4 \%$ suffered from CVA and $1.8 \%$ died during TAVI hospitalisation. TTE images were reviewed by a sonographer and cardiologist and images were rated in terms of quality. Procedural echo was excellent in $2.5 \%$, good in $48.9 \%$ and poor in 10.4\%. Pre procedure echo images were excellent in $5.4 \%$ and poor in $8.3 \%$. There was no difference in the rate of poor image quality for pre procedural TTE vs procedural TTE, and poor pre-procedure TTE did not predict poor procedural image quality. 24-hour echo was performed by the same sonographer and was noted to have excellent image quality in $6.5 \%$, good in $59.7 \%$ and poor in $4 \%$. Parasternal TTE images were optimal in $79.5 \%$ of cases, apical images were optimal in $61.5 \%$ of cases and stent depth was visualised optimally in $93.5 \%$ of cases. Patients with a body surface area $>2 \mathrm{~m} 2$ were more likely to have a poor rated image as well as suboptimal apical and parasternal views. TTE and invasive haemodynamics were used to identify the need for post deployment stent ballooning due to PVL in 8.3\%. Left ventricular outflow tract and midventricular obstruction were correctly identified in 7 cases and treated. No conversion to TOE was required for image quality. Colour flow doppler quality was optimal for $82.7 \%$ and no more than mild PVL was seen in $91.4 \%$. 2 cases could not be excluded for PVL due to poor image quality, but other forms of assessment were used such as fluoroscopy, aortic root injection, and invasive haemodynamics. Poor image quality was not associated with moderate PVL. [14] Zaouter et al observed the outcomes echocardiography findings of patients receiving TAVI with either CS or GA. With the degree of PVL being assessed in GA 
patients via TOE and in CS patients via haemodynamics measure and fluoroscopy. Procedural success was similar between groups. The incidence of moderate to severe PVL after implantation was rare. There was no difference in the incidence of moderate to severe PVL after the procedure and at 5 days between the groups. 5 patients died during the procedure with 3 cases in the GA group and 10 patients died during hospital stay with 6 in the GA group and 4 in the CS group. In hospital mortality was greater in the GA group, however there was no difference at 30 days and 1 year. [15] Sherifi et al studied the echo findings of patients undergoing TAVI. Patients were sedated and had either a TOE (48 patients) or TTE (96 patients). Groups were not different in terms of patient characteristics such as age, BMI or co-morbidities. Device success was $98 \%$ in the TOE group and $94 \%$ in the TTE group. The median procedure time, fluoroscopy time and rate of procedural acute kidney injury was lower in the TOE group. There was no statistical difference in post TAVI AR in both groups. There were 3 complications detected early in the TOE group and managed successfully. [16]

\section{Considerations}

Prior to performing TAVI considerable assessment and planning is required by the anaesthetic team and cardiologists with pre-, peri-, and post-operative phases to consider. Firstly, prophylactic antibiotics should be discussed between the teams and a suitable choice shown be given whilst checking for allergies and ensuring renal and liver function are stable and appropriate for the given antibiotic dose. If GA is chosen; invasive monitoring such as an arterial line and CVC should be used, which can come with complications such as internal carotid artery cannulation, pneumothorax, bleeding, infection though this is minimised due to ultrasound guidance and operator experience. Choice of anaesthetic agents for induction is dependent of the anaesthetists familiarly and experience of the drugs; typically, propofol, midazolam, fentanyl, ketamine can all be used during induction. These agents have a multitude of side effects such as respiratory depression, bradycardia, and haemodynamic collapse resulting in the need for vasopressors. Neuromuscular blockade is required in these cases and come with their own set of side effects such as rocuronium which has been favourable for rapid sequence induction and has been known to cause anaphylaxis. Advantages of GA however are multiple: definite airway control, patient compliance, easier management of haemodynamic challenges as well as ventilating patients who cannot remain supine for long periods such as those with sleep apnoea. [2]

Bispectral index (BIS) is a useful measure of the depth of anaesthesia, in conjunction with the patient's clinical status and external factors such as drugs, temperature, volume status can be used to assess the depth of anaesthesia. BIS provide a numerical value which and can help balance the quantity of anaesthetic agents to reach a certain depth of sedation and haemodynamic stability. He et al performed a study looking into the effectiveness of BIS in patients with sedation. In the sedation group low doses of dexmetomidine, propofol, and remifentanyl were infusion targeting a BIS range of 60-70 with addition boluses of propofol or fentanyl to decrease BIS to 40-60 during operator activity such as skin incision. Of 117 patients 77 had BIS monitoring for TAVI under sedation and LA. The GA group and Sedation group has similar patients' characteristics. Total procedure time was less in the sedation group, with less blood loss and less catecholamine use. Rate of complications such as ventricular fibrillation and cardiac tamponade was similar between groups. 2 patients were converted to GA. 30-day mortality was similar between groups and length of hospital stay was longer in the GA group. Post-operative nausea and vomiting were less in the sedation group, and the GA group has a higher rate of pulmonary infection and pleural effusion. [17]

A case may be made for sedation over GA in terms of cost effectiveness, with finance influencing decisions being made in modern medical institutions as long as patient safety is not compromised; Toppen et al studied the clinical and financial outcomes of patients undergoing TAVI using CS. 196 patients were included in the study with 147 having GA and 49 having CS. In hospital mortality was $1.5 \%$ with a trend towards lower mortality in the CS group. The frequency of major adverse events was similar between groups. CS patients had fewer ICU hours, hospital days, and for 30 day follow up had improvement quality of life questionnaire scores. 30-day readmission rates were not different between groups. Costs for the CS groups were lower in multiple categories, such as ICU cost, anaesthesia Cost, operating room recovery cost, pharmacy cost, room 
cost. [18] Ahmad et al performed a retrospective review 418 patients with $46.4 \%$ having CS. Patients having GA were likely to be older, have symptomatic heart failure and lower average pre-procedural haemoglobin. Patients receiving CS had shorted ICU and hospital stays. In terms of costs however there was no statistically significant difference between the groups, with average total, direct and indirect cost being slightly higher in the CS group. Though CS was cost effective in terms of pharmacy cost, room cost, supply, physical therapy and imaging. [19] Mosleh et al performed a propensity matched analysis comparing patients undergoing TAVI with either GA or CS with 154 patients each. There was no difference in in hospital safety outcomes, 30-day mortality, or 30-day stroke between groups. They found shorter procedural times, length of stay, ICU stay and lower direct costs in every departmental category. [20]

\section{Complications, and Drawbacks}

When using LA; routine monitoring is required, though the need for invasive monitoring is at anaesthetist's discretion. Sedation with similar agents as in GA such as propofol, midazolam can even at lower doses result in the side effects such as respiratory depression, loss of airway reflexes and haemodynamic compromise especially in the elderly who are more sensitive to these agents. Patients can vary in their depth of anaesthesia which can be challenging given a lack of definitive airway and require constant monitoring and observation by the anaesthetists. Risk of LA can include LA toxicity, due to accidental intravascular infiltration of LA. Though this is uncommon due to ultrasound use, good technique, and operator experience. [21] Mayr et al suggest the presence of anaesthetic support in use of CS in TAVI. 2009 patients underwent transfemoral TAVI between 2011 and 2016. 30\% had CS with critical adverse events noted in 9\%. CPR was performed in $2 \%$ and defibrillation in $1.6 \%$. Conversion to GA occurred in 24 patients with $2 \%$ due to respiratory distress and $1 \%$ due to pain. Catecholaminergic support was required in $45 \%$ with $41 \%$ receiving vasopressors. [22]

Goldfuss et al studied the anaesthetic related complications during TAVI. 853 underwent GA with appropriate invasive monitoring, pre oxygenation and induction of etomidate, rocuronium and an infusion of remifentanyl. Inhalational anaesthetic agents such as sevoflurane were for maintenance. Mean age was $79+/-6$ years with mean BMI $27+/-5 \mathrm{~kg} / \mathrm{m} 2$. Most patients had an American Society of Anaesthesiologists (ASA) score of 3. Transfemoral (TF) access was used in $59.3 \%$ and transapical (TA) in $40.7 \%$. Common complications included difficult intubations $(0.7 \%)$, airway damage $(0.9 \%)$, Dental damage $(0.4 \%)$, laryngospasm $(0.2 \%)$ and allergic reactions $(0.4 \%)$. Despite $17.5 \%$ receiving prophylaxis against postoperative nausea and vomiting; 232 patients still developed symptoms. Delirium occurred in 74 patients with factors such as male gender, and pre-existing dementia influencing delirium rate. $44 \%$ were hypothermic by $0.8+/-1.1$ degrees though postoperative complicate rate nor mortality rate were significantly increased in this group. 75 patients were noted to be hypoxaemic during the start of anaesthesia with 22 patients with severe hypoxaemia. $61.3 \%$ of these patients received midazolam as premedication. [23]

Cerebral perfusion can be compromised at two points during TAVI: on induced cardiac arrest for balloon valvuloplasty and hypotension during valve release. Which in turn can affect post-operative morbidity and mortality as well as neurocognitive function. Anaesthetic agents can also affect haemodynamics and impair cerebral circulation. Mayr et al compared cerebral oxygen saturation and neurocognitive outcomes in patients receiving GA and sedation in the INSERT trial. Cerebral oximetry was monitored by near infrared spectroscopy. In 66 patients (31 in GA and sedation groups each) with sedation being carried out with an infusion of propofol $1 \mathrm{mg} / \mathrm{kg} / \mathrm{hr}$ and remifentanyl $0.03 \mathrm{mcg} / \mathrm{kg} / \mathrm{hr}$ with $10 \mathrm{ml}$ of $1 \%$ Mepivacaine infiltrated to skin aiming for moderate to DS. 39\% experienced peri-operative cerebral desaturation below the desaturation threshold, though there was no significant difference between groups. Neurocognition was assessed by memory, speech fluency and executive function which revealed no significant difference preor post-operative between groups. Procedure time, induction time, time taken to transport to ICU were comparable between groups. ICU stay was shorter in the GA group. Patients in the sedation group had a higher rate of respiratory events and need for bag valve mask ventilation (19\%). Unrest and pain (61\%) were also noted in the sedation group. $94 \%$ of sedation patients developed adverse events as oppose to $13 \%$ in the GA group with one patient (sedation group) developing a peri-operative stroke without signs of cerebral desaturation. Type of anaesthesia used did not affect implant outcome. [24] 
Delirium is a common disturbance of cerebral function which can be seen in the elderly, frail, and patients with multiple co-morbidities. Postoperative delirium (POD) can be because of one or more pathophysiological stressors such as major surgery, sepsis, hypotension, GA and can increase the risk of mortality in these patients. Van der Wulp et al in a single centre study observed the outcomes of patients who underwent GA for a TAVI and the occurrence of POD. Of 703 TAVI patients with POD observed in 16.5\%. POD occurred a median of 1 day after TAVI and continued for a median of 3 days. Mean age was 82 years in the POD groups and $49.5 \%$ were male. $25.9 \%$ of patients who developed POD had a history of delirium. 30-day survival was significantly lower in patients with POD. Long term follow up showed impaired 1 year, 3 year and 5 year survival in patients with POD. Complications such as CVA, infection, and atrial fibrillation were more frequently observed in patients with POD and longer hospital stays were noted. Patients with POD were older, had less body mass index, and a smaller aortic valve area. Device success was seen in 93.6\%. There was also no difference in procedural duration between groups. [25] Goudzwaard et al studied the indicators and outcomes of TAVI patients with POD. 543 patients were enrolled with $43 \%$ having GA. Mean age was $70.1+/-8$ years and $55 \%$ were men. There were no patients with a diagnosis of dementia. Incidence of delirium was $14 \%$ and patients who developed POD tended to be older, had a high prevalence of renal failure and stroke, and were considered frail. 59\% of POD patients had GA with GA, non-transfemoral access and longer procedure time being associated with POD. [26]

\section{Conclusion}

The use of sedation with or without local anaesthesia appears to be a safe and effective method to facilitate TAVI. The use of sedation over general anaesthesia has been shown to reduce length of stay in the ICU and in hospital, reduce polypharmacy and cause much less haemodynamic compromise. In those who are high risk; frail, elderly or have severe co-morbidities the risk of GA may be too great and can outweigh the benefits intended from TAVI. Experienced centres will have clinicians trained in how to anaesthetise and deal with these group of patients; a multidisciplinary team discussion is vital to ensure the appropriate anaesthetic is chosen for each patient. As TAVI continues to revolutionise the treatment for symptomatic aortic stenosis, the rise in cases in inevitable and the use of sedation and local anaesthesia allows clinicians with a viable option to treat a challenging group of patients.

References:

1) Imnadze G, Hofmann S, Billion M, et al. Clinical value of the $20 \%$ logistic EuroSCORE cut-off for selecting TAVI candidates: a single-centre cohort study analysis. Open Heart . 2020;7(1):e001194. Published 2020 Feb 19. doi:10.1136/openhrt-2019-001194

2) Afshar AH, Pourafkari L, Nader ND. Periprocedural considerations of transcatheter aortic valve implantation for anesthesiologists. J Cardiovasc Thorac Res . 2016;8(2):49-55. doi:10.15171/jcvtr.2016.10

3) Holmes DR Jr, Mack MJ, Kaul S, Agnihotri A, Alexander KP, Bailey SR, Calhoon JH, Carabello BA, Desai MY, Edwards FH, Francis GS, Gardner TJ, Kappetein AP, Linderbaum JA, Mukherjee C, Mukherjee D, Otto CM, Ruiz CE, Sacco RL, Smith D, Thomas JD. 2012 ACCF/AATS/SCAI/STS expert consensus document on transcatheter aortic valve replacement. J Am Coll Cardiol. 2012 Mar 27;59(13):1200-54. doi: 10.1016/j.jacc.2012.01.001. Epub 2012 Jan 31. PMID: 22300974.

4) Debry N, Delhaye C, Azmoun A, Ramadan R, Fradi S, Brenot P, Sudre A, Moussa MD, Tchetche D, Ghostine S, Mylotte D, Modine T. Transcarotid Transcatheter Aortic Valve Replacement: General or Local Anesthesia. JACC Cardiovasc Interv. 2016 Oct 24;9(20):2113-2120. doi: 10.1016/j.jcin.2016.08.013. PMID: 27765304

5) Gauthier C, Astarci P, Baele P, et al. Mid-term survival after transcatheter aortic valve implantation: Results with respect to the anesthetic management and to the access route (transfemoral versus transapical). Ann Card Anaesth . 2015;18(3):343-351. doi:10.4103/0971-9784.159804

6) Renner J, Tesdorpf A, Freitag-Wolf S, et al. A retrospective study of conscious sedation versus general anaesthesia in patients scheduled for transfemoral aortic valve implantation: A single center experi- 
ence. Health Sci Rep . 2018;2(1):e95. Published 2018 Nov 1. doi:10.1002/hsr2.95

7) Husser O, Fujita B, Hengstenberg C, Frerker C, Beckmann A, Möllmann H, Walther T, Bekeredjian R, Böhm M, Pellegrini C, Bleiziffer S, Lange R, Mohr F, Hamm CW, Bauer T, Ensminger S; GARY Executive Board. Conscious Sedation Versus General Anesthesia in Transcatheter Aortic Valve Replacement: The German Aortic Valve Registry. JACC Cardiovasc Interv. 2018 Mar 26;11(6):567-578. doi: 10.1016/j.jcin.2017.12.019. PMID: 29566803.

8) Miles LF, Joshi KR, Ogilvie EH, Densem CG, Klein AA, O'Sullivan M, Martinez G, Sudarshan CD, AbuOmar Y, Irons JF. General anaesthesia vs. conscious sedation for transfemoral aortic valve implantation: a single UK centre before-and-after study. Anaesthesia. 2016 Aug;71(8):892-900. doi: 10.1111/anae.13522. Epub 2016 Jun 29. PMID: 27353456.

9) Goren O, Finkelstein A, Gluch A, Sheinberg N, Dery E, Matot I. Sedation or general anesthesia for patients undergoing transcatheter aortic valve implantation-does it affect outcome? An observational single-center study. J Clin Anesth. 2015 Aug;27(5):385-90. doi: 10.1016/j.jclinane.2015.03.025. Epub 2015 Apr 24. PMID: 25912486.

10) Thiele H, Kurz T, Feistritzer HJ, Stachel G, Hartung P, Lurz P, Eitel I, Marquetand C, Nef H, Doerr O, Vigelius-Rauch U, Lauten A, Landmesser U, Treskatsch S, Abdel-Wahab M, Sandri M, Holzhey D, Borger M, Ender J, Ince H, Öner A, Meyer-Saraei R, Hambrecht R, Fach A, Augenstein T, Frey N, König IR, Vonthein R, Rückert Y, Funkat AK, Desch S, Berggreen AE, Heringlake M, de Waha-Thiele S; SOLVETAVI Investigators. General Versus Local Anesthesia With Conscious Sedation in Transcatheter Aortic Valve Implantation: The Randomized SOLVE-TAVI Trial. Circulation. 2020 Oct 13;142(15):1437-1447. doi: 10.1161/CIRCULATIONAHA.120.046451. Epub 2020 Aug 21. PMID: 32819145.

11) Romano SM, Ristalli F, Giglioli C, et al. Deep sedation vs femoral block anesthesia: beat-by-beat hemodynamic impact on TAVI procedure. Am J Cardiovasc Dis . 2020;10(4):340-349. Published 2020 Oct 15.

12) Durand E, Borz B, Godin M, Tron C, Litzler PY, Bessou JP, Bejar K, Fraccaro C, Sanchez-Giron C, Dacher JN, Bauer F, Cribier A, Eltchaninoff H. Transfemoral aortic valve replacement with the Edwards SAPIEN and Edwards SAPIEN XT prosthesis using exclusively local anesthesia and fluoroscopic guidance: feasibility and 30-day outcomes. JACC Cardiovasc Interv. 2012 May;5(5):461-467. doi: 10.1016/j.jcin.2012.01.018. Epub 2012 May 9. PMID: 22560979.

13) Oguri A, Yamamoto M, Mouillet G, Gilard M, Laskar M, Eltchaninoff H, Fajadet J, Iung B, DonzeauGouge P, Leprince P, Leguerrier A, Prat A, Lievre M, Chevreul K, Dubois-Rande JL, Chopard R, Van Belle E, Otsuka T, Teiger E; FRANCE 2 Registry Investigators. Clinical outcomes and safety of transfemoral aortic valve implantation under general versus local anesthesia: subanalysis of the French Aortic National CoreValve and Edwards 2 registry. Circ Cardiovasc Interv. 2014 Aug;7(4):602-10. doi: 10.1161/CIRCINTERVENTIONS.113.000403. Epub 2014 Jul 8. PMID: 25006175

14) Jain R, O'Hair DP, Bajwa TK, et al. Transthoracic echocardiography is adequate for intraprocedural guidance of transcatheter aortic valve implantation. Echo Res Pract . 2017;4(4):63-72. doi:10.1530/ERP-170050

15) Zaouter C, Smaili S, Leroux L, Bonnet G, Leuillet S, Ouattara A. Transcatheter aortic valve implantation: General anesthesia using transesophageal echocardiography does not decrease the incidence of paravalvular leaks compared to sedation alone. Ann Card Anaesth . 2018;21(3):277-284. doi:10.4103/aca.ACA_204_17

16) Sherifi I, Omar AMS, Varghese M, et al. Comparison of transesophageal and transthoracic echocardiography under moderate sedation for guiding transcatheter aortic valve replacement. Echo Res Pract . 2018;5(2):79-87. doi:10.1530/ERP-17-0080

17) He W, Huang RR, Shi QY, Liu XB, Wang JA, Yan M. Bispectral index-guided sedation in transfemoral 
transcatheter aortic valve implantation: a retrospective control study. J Zhejiang Univ Sci B . 2017;18(4):353359. doi:10.1631/jzus.B1600522

18) Toppen W, Johansen D, Sareh S, et al. Improved costs and outcomes with conscious sedation vs general anesthesia in TAVR patients: Time to wake up? PLoS One . 2017;12(4):e0173777. Published 2017 Apr 5. doi:10.1371/journal.pone. 0173777

19) Ahmad M, Patel JN, Vipparthy SC, et al. Conscious Sedation Versus General Anesthesia in Transcatheter Aortic Valve Replacement: A Cost and Outcome Analysis. Cureus . 2019;11(6):e4812. Published 2019 Jun 3. doi: $10.7759 /$ cureus. 4812

20) Mosleh W, Mather JF, Amer MR, Hiendlmayr B, Kiernan FJ, McKay RG. Propensity Matched Analysis Comparing Conscious Sedation Versus General Anesthesia in Transcatheter Aortic Valve Implantation. Am J Cardiol. 2019 Jul 1;124(1):70-77. doi: 10.1016/j.amjcard.2019.03.042. Epub 2019 Apr 10. PMID: 31064667.

21) Melidi E, Latsios G, Toutouzas K, Vavouranakis M, Tolios I, Gouliami M, Gerckens U, Tousoulis D. Cardio-anesthesiology considerations for the trans-catheter aortic valve implantation (TAVI) procedure. Hellenic J Cardiol. 2016 Nov-Dec;57(6):401-406. doi: 10.1016/j.hjc.2016.10.001. Epub 2016 Oct 6. PMID: 28375080 .

22) Mayr NP, Wiesner G, Husser O, Joner M, Michel J, Knorr J, Pellegrini C, Bleiziffer S, Schunkert H, Lange R, Tassani-Prell P. Critical adverse events during transfemoral TAVR in conscious sedation. Is an anesthesiologic support mandatory? Cardiovasc Revasc Med. 2018 Sep;19(6S):41-46. doi: 10.1016/j.carrev.2018.06.014. Epub 2018 Jun 21. PMID: 30327095.

23) Goldfuss S, Wittmann S, Würschinger F, et al. Anaesthesia-related complications and side-effects in TAVI: a retrospective study in Germany. BMJ Open . 2019;9(4):e025825. Published 2019 May 1. doi:10.1136/bmjopen-2018-025825

24) Mayr NP, Hapfelmeier A, Martin K, Kurz A, van der Starre P, Babik B, Mazzitelli D, Lange R, Wiesner G, Tassani-Prell P. Comparison of sedation and general anaesthesia for transcatheter aortic valve implantation on cerebral oxygen saturation and neurocognitive outcome+. Br J Anaesth. 2016 Jan;116(1):90-9. doi: 10.1093/bja/aev294. Epub 2015 Sep 29. PMID: 26424178.

25) van der Wulp K, van Wely M, van Heijningen L, et al. Delirium After Transcatheter Aortic Valve Implantation Under General Anesthesia: Incidence, Predictors, and Relation to Long-Term Survival. J Am Geriatr Soc . 2019;67(11):2325-2330. doi:10.1111/jgs.16087

26) Goudzwaard JA, de Ronde-Tillmans MJAG, de Jager TAJ, et al. Incidence, determinants and consequences of delirium in older patients after transcatheter aortic valve implantation. Age Ageing . 2020;49(3):389-394. doi:10.1093/ageing/afaa001

27) Yamamoto M, Meguro K, Mouillet G, Bergoend E, Monin JL, Lim P, Dubois-Rande JL, Teiger E. Effect of local anesthetic management with conscious sedation in patients undergoing transcatheter aortic valve implantation. Am J Cardiol. 2013 Jan 1;111(1):94-9. doi: 10.1016/j.amjcard.2012.08.053. Epub 2012 Oct 12. PMID: 23068861.

28) Hyman MC, Vemulapalli S, Szeto WY, Stebbins A, Patel PA, Matsouaka RA, Herrmann HC, Anwaruddin S, Kobayashi T, Desai ND, Vallabhajosyula P, McCarthy FH, Li R, Bavaria JE, Giri J. Conscious Sedation Versus General Anesthesia for Transcatheter Aortic Valve Replacement: Insights from the National Cardiovascular Data Registry Society of Thoracic Surgeons/American College of Cardiology Transcatheter Valve Therapy Registry. Circulation. 2017 Nov 28;136(22):2132-2140. doi: 10.1161/CIRCULATIONAHA.116.026656. Epub 2017 Sep 1. PMID: 28864443.

29) Bergmann L, Kahlert P, Eggebrecht H, Frey U, Peters J, Kottenberg E. Transfemoral aortic valve implantation under sedation and monitored anaesthetic care-a feasibility study. Anaesthesia. 2011 Nov;66(11):97782. doi: 10.1111/j.1365-2044.2011.06788.x. Epub 2011 Sep 20. PMID: 21933156. 


\section{Hosted file}

Table 1.docx available at https://authorea.com/users/427600/articles/531776-transcatheteraortic-valve-implantation-tavi-is-general-anaesthesia-a-necessity

\section{Hosted file}

Table 2.docx available at https://authorea.com/users/427600/articles/531776-transcatheteraortic-valve-implantation-tavi-is-general-anaesthesia-a-necessity

\section{Hosted file}

Table 3.docx available at https://authorea.com/users/427600/articles/531776-transcatheteraortic-valve-implantation-tavi-is-general-anaesthesia-a-necessity 